\title{
A prescrição de medicação psicotrópica e o conhecimento da portaria regulatória brasileira por cirurgiões-dentistas
}

\author{
The prescription of psychotropic medication and the \\ knowledge of brazilian regulatory legislation by dental surgeons
}

\author{
Luanne Priscilla Pereira Avelar ${ }^{1}$ (D), Lia Silva de Castilho² (D), \\ Mauro Henrique Nogueira Guimarães Abreu $^{3}$ (D), Ênio Lacerda Vilaça² (D), \\ Vera Lúcia Silva Resende ${ }^{2}$ (D), Maria Elisa Souza e Silva² (D)
}

\begin{abstract}
Resumo
Introdução: O uso de psicotrópicos por cirurgiões-dentistas é permitido por lei no Brasil para casos clínicos específicos. São poucos os estudos sobre a sua utilização na odontologia. Objetivo: Este estudo investigou a prescrição de psicotrópicos por cirurgiões-dentistas inscritos no Conselho Regional de Minas Gerais e seu conhecimento sobre a Portaria 344/1998. Método: Foram enviados aos 15.250 cirurgiões-dentistas um questionário eletrônico com 13 questões abordando a prescrição de psicotrópicos, e foram respondidos 969 questionários. Foram estudados: o tempo de formado, tipo de instituição de graduação, tipo de pós-graduação, medicamentos mais prescritos, conhecimento sobre a Portaria 344/1998 e público mais frequente que recebe a prescrição. Resultados: 257 profissionais prescrevem psicotrópicos e 223 conhecem a legislação. Um preocupante percentual de profissionais que prescreve esses medicamentos não conhece o teor da Portaria 344/1998. Os analgésicos opioides são os medicamentos mais prescritos. Os especialistas são os que mais prescrevem psicotrópicos $(p=0,015)$, e aqueles que prescrevem ansiolíticos conhecem a legislação com maior frequência $(p=0,003)$. Os cirurgiões bucomaxilofaciais $(p=0,02)$ e os especialistas em distúrbios da articulação temporomandibular $(0,03)$ são os que mais conhecem a legislação e prescrevem medicação psicotrópica. A dor forte e o medo são as duas principais razões para a prescrição. Conclusão: Ser especialista e conhecer a Portaria 344/1998 são fatores associados à prescrição de psicotrópicos entre cirurgiões-dentistas.
\end{abstract}

Palavras-chave: psicotrópicos; medicamentos sob prescrição; odontologia.

\begin{abstract}
Background: The use of psychotropics by dental surgeons is permitted by law in Brazil for specific clinical cases. There are few studies on its use in dentistry. Objective: This study investigated the prescription of psychotropics drugs by dentists enrolled in the Regional Council of Minas Gerais and their knowledge about regulatory ordinance 344/1998. Method: An electronic questionnaire with 13 questions addressing the prescription of psychotropic drugs was sent to 15250 dental surgeons and 969 questionnaires were answered. It was considered the time of graduation, type of undergraduate institution, type of postgraduate course, more frequent prescribed medication, knowledge about ordinance 344/1998 and more frequent public that receives the prescription. Results: Two hundred and fifty-seven professionals prescribe psychotropic drugs and $223 \mathrm{know}$ the law, and a worrying percentage
\end{abstract}

'Graduação em Odontologia, Faculdade de Odontologia, Universidade Federal de Minas Gerais (UFMG) - Belo Horizonte (MG), Brasil. ${ }^{2}$ Departamento de Odontologia Restauradora, Faculdade de Odontologia, Universidade Federal de Minas Gerais (UFMG) - Belo Horizonte (MG), Brasil. ${ }^{3}$ Departamento de Odontologia Social e Preventiva, Faculdade de Odontologia, Universidade Federal de Minas Gerais (UFMG) - Belo Horizonte (MG), Brasil. Trabalho realizado na Faculdade de Odontologia, Universidade Federal de Minas Gerais (UFMG) e Conselho Regional de Odontologia de Minas Gerais - Belo Horizonte (MG), Brasil.

Endereço para correspondência: Luanne Priscilla Pereira Avelar - Graduação em Odontologia, Faculdade de Odontologia, Universidade Federal de Minas Gerais (UFMG), Av. Presidente Antônio Carlos, 6627 - Pampulha - CEP: 31270-000 - Belo Horizonte (MG), Brasil - Email: Iuannepriscilla1@hotmail.com

Fonte de financiamento: nenhuma.

Conflito de interesses: nada a declarar.

Este é um artigo publicado em acesso aberto (Open Access) sob a licença Creative Commons Attribution, que permite uso, distribuição e reprodução em qualquer meio, sem restrições desde que o trabalho original seja corretamente citado. 
of professionals who prescribe these drugs does not know the content of the Ordinance 344/1998. Opioid analgesics are the most prescribed psychotropic medications. Specialists are the ones who prescribe psychotropic drugs $(\mathrm{p}=0.015)$ and those professionals who prescribe anxiolytics know the legislation more frequently $(p=0.003)$. The maxillofacial surgeons $(p=0.02)$ and experts in disorders of the temporomandibular jaw joint $(0.03)$ are the ones who know the law and prescribe psychotropic medication. Strong pain and fear are the two main reasons for prescribing psychotropic drugs.

Conclusion: Being a specialist and knowing the ordinance 344/1998 are factors associated with the prescription of psychotropic medications among dentists.

Keywords: psychotropic drugs; prescription drugs; dentistry.

\section{INTRODUÇÃO}

Segundo a Organização Mundial da Saúde (OMS), psicofármacos, ou drogas psicotrópicas, são substâncias psicoativas que agem diretamente no Sistema Nervoso Central ${ }^{1}$. Nesse grupo de medicamentos estão incluídos os analgésicos opioides, os ansiolíticos, os antidepressivos, os antipsicóticos e os anticonvulsivantes. Esses medicamentos têm sua comercialização regulada pela Portaria 344/1998. Através dessa portaria, foi legalizada a prescrição de medicamentos entorpecentes e psicotrópicos por cirurgiões-dentistas, para fins odontológicos².

No exercício da clínica odontológica, o cirurgião-dentista (CD) pode se deparar com casos de pacientes extremamente ansiosos em relação ao tratamento odontológico e/ou com um baixo nível cognitivo, que os leva a não cooperar para o sucesso da realização do tratamento. Nessas situações, o uso de medicamentos psicotrópicos pode ser de grande auxílio, tanto para o paciente quanto para o profissional ${ }^{3}$.

Os analgésicos opioides são utilizados em odontologia em casos de dores agudas e fortes ${ }^{4}$. Os benzodiazepínicos são os ansiolíticos de primeira escolha utilizados para controle da ansiedade e medo, por serem eficazes e terem margem de segurança clínica ampla ${ }^{5}$. Os anticonvulsivantes e antidepressivos tricíclicos são indicados especificamente para tratamento de dores crônicas temporomandibulares. Os anticonvulsivantes são utilizados para tratamento de dor neuropática, associada à lesão trigeminal, neuralgia pós-herpética e nevralgia diabética ${ }^{6}$. Os antidepressivos são empregados em casos de cefaleia, dor muscoloesquelética e algumas desordens de dor neuropática ${ }^{7}$, no tratamento do bruxismo do sono $^{8}$ e no tratamento da síndrome da ardência bucal, assim como os antipsicóticos e benzodiazepínicos?.

O padrão de prescrição dessas substâncias varia muito entre os países. A prescrição de medicamentos controlados por cirurgiões-dentistas clínicos-gerais no país de Gales é mínima, quando comparada ao total de prescrições odontológicas ${ }^{10}$. Nos Estados Unidos, entretanto, as prescrições da associação entre paracetamol e hidrocodona são frequentes, particularmente para cirurgias de terceiros molares inclusos ${ }^{11}$. Na Austrália, o total das prescrições de associações de paracetamol e codeína também são frequentes e superam as prescrições do ibuprofeno ${ }^{12}$. No Brasil, de um total de 16.436 prescrições de ansiolíticos, sedativos e hipnóticos, por cirurgiões-dentistas no ano de 2010, 64,30\% eram ansiolíticos e 35,70\% eram sedativos e hipnóticos ${ }^{5}$.

Além disso, o total de prescrições desses medicamentos vem aumentando. Na Austrália, de 2001 a 2012, a prescrição geral de benzodiazepínicos aumentou 47\%, com aumento médio anual de 3,9. Em relação aos opioides, as prescrições desses medicamentos aumentaram na prática odontológica em $344 \%$ e $184 \%$ respectivamente ${ }^{12}$.

A despeito dos benefícios da utilização de psicotrópicos em certas situações clínicas, é imprescindível analisar os hábitos de prescrição desses medicamentos por cirurgiões-dentistas, para identificar problemas, auxiliar na implementação de medidas preventivas e corretivas ${ }^{5,10-14}$, além de prevenir a ocorrência de efeitos adversos dos medicamentos ${ }^{12}$. Para prescrever de forma satisfatória os medicamentos psicotrópicos, é preciso conhecer a legislação brasileira. Dessa forma, são evitadas prescrições em formulários indevidos com consequente negativa da dispensação farmacêutica.

Existem poucos estudos sobre a prescrição de medicamentos no Brasil e a sua utilização na prática clínica odontológica, principalmente sobre prescrição de psicofármacos por cirurgiões-dentistas. Assim, este estudo tem por objetivo investigar a prescrição de psicotrópicos por cirurgiões-dentistas inscritos no Conselho Regional de Minas Gerais e seu conhecimento sobre a Portaria 344/1998. A hipótese do presente estudo é que o conhecimento da legislação reguladora da prescrição de psicofármacos por cirurgiões-dentistas do Estado de Minas Gerais está associado ao ato de prescrever esses medicamentos.

\section{MÉTODO}

Estudo observacional e transversal que foi aprovado pelo Comitê de Ética e Pesquisa da Universidade Federal de Minas Gerais (UFMG) por meio do parecer no 1.302 .991 (CAAE no 49765515.0.0000.5149).

A coleta de informações individualizadas foi realizada através de questionário eletrônico respondido voluntariamente por cirurgiões-dentistas do Estado de Minas Gerais que possuíam e-mail atualizado no Conselho Regional de Odontologia de Minas Gerais (CROMG), em março de 2016. Do total de 25.416 dentistas vinculados à instituição, 15.250 e-mails estavam disponíveis. O termo de esclarecimento livre e esclarecido foi disponibilizado 
a todos e o profissional pôde optar por participar ou não da pesquisa.

Para garantir a confidencialidade dos dados dos profissionais vinculados ao CROMG, os questionários eletrônicos foram enviados pela própria instituição aos CDs que possuíam o e-mail atualizado. Após 30 dias, em função do número de respostas ter sido pouco expressivo, o questionário eletrônico foi novamente encaminhado. Desta vez, foram realizadas alterações na estratégia de envio dos e-mails, a fim de se evitar que fossem considerados spams ou direcionados para a lixeira. O CROMG foi o responsável pelo envio dos e-mails, porém o remetente foi vinculado a um e-mail pessoal de uma das pesquisadoras. Após 60 dias do envio do primeiro e-mail, obteve-se o retorno de 986 questionários, sendo que 969 profissionais aceitaram participar da pesquisa e 17 se recusaram (1,72\%).

O questionário foi criado pelos pesquisadores na plataforma Google Forms, aplicativo disponível no Google Docs, um serviço do Google que permite a edição colaborativa de documentos de forma simples e organizada. Esse questionário contemplou 13 perguntas, sendo o profissional guiado para a próxima questão de acordo com a resposta anterior.

As perguntas sobre a caracterização da amostra versaram sobre o tempo de formado, instituição de graduação e grau de escolaridade. As questões seguintes abordaram o conhecimento sobre a portaria ministerial que concede ao $\mathrm{CD}$ a prerrogativa de prescrever psicofármacos, se o CD prescrevia psicofármacos para seus pacientes, sobre os motivos para a prescrição ou não desses medicamentos, sobre a frequência de prescrição para cada classe de medicamentos (analgésico opioide, ansiolíticos, antidepressivos, anticonvulsivantes e antipsicóticos), a faixa etária dos pacientes para os quais as prescrições eram destinadas e frequência de vezes que se realizava prescrições de psicofármacos. Para a análise da frequência, as respostas à questão "Qual é a frequência que você prescreve o medicamento?" foram assim agrupadas: "Muito" e "Às vezes" foram agrupadas como "prescrição frequente" e "Raramente" e "Nunca" como "prescrição infrequente". As especialidades odontológicas que lidam mais frequentemente com as principais indicações para uso desses medicamentos e que foram analisadas separadamente foram escolhidas a partir da revisão da literatura ${ }^{6-9}$.

Apesar de o instrumento não ter sido formalmente validado, para avaliação da clareza das perguntas, antes da coleta principal, ele foi aplicado a 30 cirurgiões-dentistas de forma presencial, após concederem sua concordância em participar da pesquisa. Passados 30 dias, o questionário foi novamente aplicado aos mesmos CDs, para validação do grau de estabilidade temporal do instrumento. $\mathrm{O}$ teste Cohen Kappa para concordância das respostas variou de 0,82 a 1,0 .

As respostas dos dentistas participantes foram tabuladas e analisadas descritivamente. A análise bivariada foi realizada para verificar possíveis associações entre conhecimento da Portaria Ministerial 344/1998, o tempo de formado e a origem da instituição de graduação e medicamentos prescritos. Os testes usados foram o teste do qui-quadro de Pearson e teste exato de Fischer. Todas as análises foram realizadas através do programa Epi Info versão 7.1.415.

\section{RESULTADOS}

A primeira mensagem eletrônica enviada foi visualizada por 1.830 cirurgiões-dentistas e 338 respostas retornaram. Já o segundo e-mail obteve 3.353 visualizações e 648 respostas foram enviadas, demonstrando que a estimulação e esclarecimentos feitos no envio do segundo e-mail foram efetivos. Do total de 15.250 e-mails enviados, 986 foram respondidos e 969 profissionais concordaram em participar da pesquisa, correspondendo a $6,3 \%$ de taxa de retorno.

Do total de 969 cirurgiões-dentistas, a média de tempo de formado foi de 16 anos $( \pm 10,11)$, com variação entre 1 e 57 anos de formação. Não houve associação entre o tempo de formado e o conhecimento sobre a Portaria Ministerial para prescrição de psicotrópicos.

Quanto ao nível de qualificação profissional, 742 (76,6\%) dos profissionais possuem, no mínimo, curso de especialização em odontologia. Do restante de 227 cirurgiões-dentistas, 7,2\% deles eram especialistas em outras áreas e 204 profissionais não possuem curso de especialização, mestrado ou doutorado em odontologia. Do total da amostra, 196 profissionais são mestres $(20,22 \%)$ e $82(8,50 \%)$ são doutores. Vinte e três mestres e doutores não possuíam especialização. Observa-se que os especialistas prescrevem mais psicofármacos que os não especialistas $(\mathrm{p}=0,015)$ (Tabela 1$)$.

Quanto ao conhecimento da legislação, as especialidades em Cirurgia e Traumatologia Bucomaxilofacial (CTBM) $(p=0,02)$ e em Distúrbios Temporomandibulares (DTM) $(\mathrm{p}=0,03)$ são as que mais relatam conhecer o teor da Portaria 344/1998 (Tabela 2).

Observa-se que quem conhece a legislação tem 2,97 mais chances de prescrever medicamentos psicotrópicos do que quem não conhece o teor da legislação $(\mathrm{p}<0,001)$. Por outro lado, é preocupante constatar que a maior parte dos CDs que prescrevem psicotrópicos desconhece o texto da Portaria Ministerial 344/1998. Em relação à origem da instituição de graduação, 51\% estudaram em instituição privada e 49\% em instituição pública. Entre aqueles que se graduaram em instituição privada, 79\% desconhecem a portaria, enquanto para os profissionais que se formaram em instituição pública, esse percentual diminui para 74\%. A associação entre o conhecimento da Portaria 344/1998 e o tipo de instituição de formação mostrou uma significância estatística marginal $(\mathrm{p}=0,05)$, confirmada pelo seu respectivo intervalo de confiança $(0,99-1,82)$. 
Tabela 1. Associação entre a prescrição de psicotrópicos por um grupo de cirurgiões-dentistas de Minas Gerais e a qualificação profissional em especialista, mestre e doutor, 2016

\begin{tabular}{|c|c|c|c|c|c|}
\hline \multirow{2}{*}{\multicolumn{2}{|c|}{ Qualificação profissional }} & \multicolumn{2}{|c|}{ Prescrição de Psicotrópicos } & \multirow{2}{*}{ OR (IC 95\%) } & \multirow{2}{*}{ Valor $\mathbf{p}$} \\
\hline & & Sim & Não & & \\
\hline \multirow[t]{2}{*}{ Especialização } & Sim & $211(28 \%)$ & $531(72 \%)$ & $\begin{array}{c}1.5 \\
(1,09-2,24)\end{array}$ & \multirow[t]{2}{*}{0,015} \\
\hline & Não & $46(20 \%)$ & $181(80 \%)$ & 1 & \\
\hline \multirow[t]{2}{*}{ Mestrado } & Sim & $53(27 \%)$ & $143(73 \%)$ & $\begin{array}{c}1,03 \\
(0,72-1,47)\end{array}$ & \multirow[t]{2}{*}{0,854} \\
\hline & Não & $204(26 \%)$ & $569(74 \%)$ & 1 & \\
\hline \multirow[t]{2}{*}{ Doutorado } & Sim & $18(22 \%)$ & $64(78 \%)$ & $\begin{array}{c}0,76 \\
(0,44-1,47)\end{array}$ & \multirow[t]{2}{*}{0,327} \\
\hline & Não & $239(27 \%)$ & $648(73 \%)$ & 1 & \\
\hline
\end{tabular}

OR = Odds Ratio; IC95\% = intervalo de confiança de 95\%; valor $\mathrm{p}$ = probabilidade de significância

Tabela 2. Associação entre a prescrição de psicofármacos e o conhecimento sobre o texto da Portaria Ministerial $344 / 1998$ e estratificação por instituição de formação e especialidades odontológicas que mais prescrevem psicofármacos e o seu conhecimento sobre o texto da Portaria Ministerial 344/1998, Minas Gerais, 2016

\begin{tabular}{|c|c|c|c|c|c|}
\hline & \multirow{2}{*}{ Prescreve } & \multicolumn{2}{|c|}{ Conhecimento sobre a legislação } & \multirow{2}{*}{ OR (IC 95\%) } & \multirow{2}{*}{ Valor de $\mathrm{p}$} \\
\hline & & Sim & Não & & \\
\hline & Sim & $99(38,52 \%)$ & $158(61,48 \%)$ & $\begin{array}{c}2,97 \\
(2,30-4,36)\end{array}$ & $<0,001$ \\
\hline & Não & $124(23,01 \%)$ & $588(76,99 \%)$ & 1 & \\
\hline \multirow{2}{*}{\multicolumn{6}{|c|}{$\begin{array}{l}\text { Especialidades Odontológicas } \\
\text { CTBMF }(\mathrm{n}=50)\end{array}$}} \\
\hline & & & & & \\
\hline & Sim & $18(53 \%)$ & $16(47 \%)$ & $\begin{array}{c}4,87 \\
(1,17-20,26)\end{array}$ & 0,02 \\
\hline & Não & $3(19 \%)$ & $13(81 \%)$ & 1 & \\
\hline \multicolumn{6}{|c|}{$\begin{array}{l}\text { Pacientes Especiais } \\
(\mathrm{n}=6)\end{array}$} \\
\hline & Sim & $2(50 \%)$ & $2(50 \%)$ & $\begin{array}{c}1 \\
(0,03-29,80)\end{array}$ & 1 \\
\hline & Não & $1(50 \%)$ & $1(50 \%)$ & 1 & \\
\hline \multicolumn{6}{|c|}{ Implantodontia } \\
\hline \multirow[t]{2}{*}{$(n=161)$} & Sim & $24(32 \%)$ & $51(68 \%)$ & $\begin{array}{c}1,36 \\
(0,68-2,71)\end{array}$ & 0,369 \\
\hline & Não & $22(25.5 \%)$ & $64(74.5 \%)$ & 1 & \\
\hline \multicolumn{6}{|c|}{ Distúrbios do sono } \\
\hline \multirow[t]{2}{*}{$(n=2)$} & Sim & $0(0 \%)$ & $0(0 \%)$ & & - \\
\hline & Não & $1(50 \%)$ & $1(50 \%)$ & & \\
\hline \multicolumn{6}{|l|}{ DTM } \\
\hline \multirow[t]{2}{*}{$(\mathrm{n}=26)$} & Sim & $7(54 \%)$ & $6(46 \%)$ & $\begin{array}{c}14 \\
(1,38-141,49\end{array}$ & 0,03 \\
\hline & Não & $1(8 \%)$ & $12(92 \%)$ & 1 & \\
\hline
\end{tabular}

OR = Odds Ratio; IC95\% = intervalo de confiança de 95\%; valor $\mathrm{p}$ = probabilidade de significância

Quando indagados sobre a existência da Portaria Ministerial 344/1998 que concede ao cirurgião-dentista a prerrogativa de prescrever medicamentos psicotrópicos, 79 (8,2\%) alegaram não ter conhecimento sobre a existência da lei e $667(68,8 \%)$ sabem da existência da legislação, mas não têm conhecimento sobre o teor do texto desta, e 223 (23\%) dos entrevistados alegaram conhecer o teor da Portaria Ministerial. Além disso, observa-se que 257 cirurgiões-dentistas, ou 26,52\%, prescrevem medicamentos psicotrópicos para os seus pacientes, mas entre estes profissionais, constatou-se que 158 (61,47\%) desconhecem o teor da portaria 344/1998 (Tabela 2).

Os medicamentos psicotrópicos mais prescritos pelos dentistas $(n=257)$ foram os analgésicos opioides $(65,36 \%$ dos casos). Ansiolíticos, hipnóticos e sedativos foram encontrados em 53,30\% dos relatos, seguidos dos antidepressivos (22,56\%), anticonvulsivantes $(15,17 \%)$ e antipsicóticos $(11,67 \%)$. Foi possível 
Tabela 3. Associação entre frequência de prescrição de cada classe de psicotrópico pelo grupo de cirurgiões-dentistas em Minas Gerais participantes do estudo e o seu conhecimento sobre o texto da Portaria Ministerial que permite ao profissional prescrever psicofármacos, 2016

\begin{tabular}{|c|c|c|c|c|c|}
\hline \multirow{2}{*}{ Droga } & \multirow{2}{*}{ Prescrição } & \multicolumn{2}{|c|}{ Conhecimento sobre Legislação } & \multirow{2}{*}{ OR (IC95\%) } & \multirow{2}{*}{ Valor $\mathbf{p}$} \\
\hline & & Sim & Não & & \\
\hline \multicolumn{6}{|l|}{ Analgésico opioide } \\
\hline & Frequente & $72(43 \%)$ & $96(57 \%)$ & $\begin{array}{c}1,72 \\
(0,99-2,97)\end{array}$ & 0,05 \\
\hline & Infrequente & $27(31 \%)$ & $62(69 \%)$ & 1 & \\
\hline \multicolumn{6}{|c|}{ Ansiolíticos, Hipnóticos e Sedativos } \\
\hline & Frequente & $64(47 \%)$ & $73(53 \%)$ & $\begin{array}{c}2,12 \\
(1,26-3,57)\end{array}$ & 0,003 \\
\hline & Infrequente & $35(29 \%)$ & $85(71 \%)$ & 1 & \\
\hline \multicolumn{6}{|l|}{ Antidepressivos } \\
\hline & Frequente & $29(50 \%)$ & $29(50 \%)$ & $\begin{array}{c}1,80 \\
(0,99-3,25)\end{array}$ & 0,05 \\
\hline & Infrequente & $71(36 \%)$ & $128(64 \%)$ & 1 & \\
\hline \multicolumn{6}{|l|}{ Antipsicóticos } \\
\hline & Frequente & $11(37 \%)$ & $19(63 \%)$ & $\begin{array}{c}0,91 \\
(0,41-2,01)\end{array}$ & 0,82 \\
\hline & Infrequente & $88(39 \%)$ & $139(61 \%)$ & 1 & \\
\hline \multicolumn{6}{|l|}{ Anticonvulsivantes } \\
\hline & Frequente & $16(41 \%)$ & $23(59 \%)$ & $1,13(0,56-2,26)$ & 0,727 \\
\hline & Infrequente & $83(38 \%)$ & $135(62 \%)$ & 1 & \\
\hline
\end{tabular}

OR = Odds Ratio; IC95\% = intervalo de confiança de 95\%; valor $\mathrm{p}=$ probabilidade de significância

constatar que, mesmo desconhecendo o teor da legislação sobre medicamentos de ação central, parte expressiva de CDs admite prescrever psicofármacos aos seus pacientes frequentemente. Para os ansiolíticos, observa-se associação entre o conhecimento da legislação e a prescrição ( $\mathrm{p}=0,003$; IC95\% 1,26-3,57). Já para os analgésicos opioides e antidepressivos, apesar de o valor de p ser igual a 0,05 , quando se observa o intervalo de confiança, verifica-se a associação não é significativa (Tabela 3 ).

Dentre as principais situações em que os cirurgiões-dentistas prescrevem psicotrópicos, dor forte, medo e agitação foram os motivos para os quais esses medicamentos são mais comumente indicados, seguidos de falta de cooperação e a pedido do paciente. Houve diferença estatisticamente significativa entre os motivos para a prescrição $(\mathrm{p}=0,000)$.

A faixa etária que mais recebe prescrição de psicotrópicos é a adulta $(96,5 \%)$, seguida de idosos $(30,7 \%)$, adolescentes $(24,1 \%)$ e crianças (14,4\%), sendo que para os três últimos a prescrição é baixa. Houve forte associação entre a prescrição de psicotrópicos e a faixa etária à qual se destinam os medicamentos $(\mathrm{p}=<0,001)$.

\section{DISCUSSÃO}

Em relação ao conhecimento da Portaria 344/1998, os presentes resultados revelam um quadro preocupante: a maioria daqueles que prescrevem essas medicações desconhece o teor da legislação. Esperar-se-ia que profissionais especialistas em CTBMF, implantodontia, odontologia para pacientes especiais, DTM e distúrbios do sono, por lidarem com situações em que os psicotrópicos podem auxiliar no tratamento odontológico, fossem mais habituados em relação à legislação regulatória da prescrição desses medicamentos em odontologia. No entanto, em todas as especialidades, há preocupante porcentagem de dentistas que prescrevem psicotrópicos mas não conhecem o conteúdo da legislação.

A ausência de associação entre o tempo de formado e o conhecimento sobre a Portaria Ministerial 344/1998 é um resultado encontrado em outras pesquisas ${ }^{16}$. Da mesma forma, o padrão de prescrição de psicotrópicos também não sofre influência desta variável, pois as variações do padrão de prescrição entre profissionais mais jovens e mais velhos são mínimas. Cirurgiões-dentistas mais velhos tendem a hábitos de prescrição mais estabelecidos. Profissionais graduados mais recentemente estão mais propensos a explorar alternativas, como inibidores da COX-2, e adotar novas estratégias terapêuticas para monitoramento das sequelas pós-operatórias ${ }^{11}$.

A maior taxa de indicação dos analgésicos opioides está de acordo com a literatura consultada ${ }^{10-12}$. Em relação às prescrições dos demais medicamentos, os presentes resultados encontraram padrão semelhante ao total geral no $\mathrm{Brasil}^{5} \mathrm{e}$ ao de outros países ${ }^{10,12}$, com exceção dos antidepressivos. Em nenhum dos estudos epidemiológicos sobre padrão de prescrição de medicamentos de ação central ${ }^{5,10-13}$ foram encontradas menções a antidepressivos. A diferença entre os percentuais de prescrição pode estar relacionada às indicações mais específicas para uso de antidepressivos, anticonvulsivantes e antipsicóticos em detrimento das indicações para o uso de ansiolíticos e analgésicos opioides em odontologia. 
A associação entre o conhecimento da Portaria 344/1998 e a prescrição de ansiolíticos pode ser explicada pelo fato de a prescrição desses medicamentos estar sujeita à Notificação de Receita Especial “B”. Na Receita Especial, o profissional deverá imprimir o talonário azul, e a Unidade da Federação concederá a numeração do talonário. Para seu uso existem regras claras sobre inscrição estadual do profissional seguindo a Instrução Normativa da Portaria 344/1998. Os demais medicamentos são dispensados pelo farmacêutico através da Receita com Controle Especial em duas vias, sendo que uma fica retida no estabelecimento comercial ${ }^{2}$. Dessa forma, o profissional que precisa prescrever através da Notificação de Receita Especial " $\mathrm{B}$ ” apresentaria um maior conhecimento do teor da Portaria $344 / 1998$ do que aqueles que não prescrevem medicamentos sob este controle.

Na Suécia, 75\% dos dentistas estudados prescrevem benzodiazepínicos para o controle da ansiedade, e $80 \%$ da amostra se considera competente para indicar o uso de tais medicamentos ${ }^{3}$. No nosso caso, os presentes resultados são preocupantes, já que é alto o percentual de indivíduos que relatam frequentemente prescrever os ansiolíticos e não conhecer o teor da Portaria 344/1998.

O baixo percentual de prescrições para crianças pode ser se justificada pelas seguintes razões: a prescrição pediátrica é mais complexa do que para adultos, visto que há diferenças fisiológicas relevantes entre os dois grupos e existem dificuldades para uma adequada administração ${ }^{17}$.

O cirurgião-dentista deve estar atento quanto às solicitações de medicamentos de ação central feitas pelos pacientes, devido aos efeitos de dependência e abstinência que esses medicamentos possuem. É comum pacientes relatarem dor crônica intratável, solicitando opiáceos aos profissionais ${ }^{7}$. Nos Estados Unidos, programas de monitoramento de prescrições de analgésicos opioides estão sendo implantados a fim de reduzir o uso abusivo desses medicamentos ${ }^{13,14}$. É por isso que talvez a prescrição de medicamentos de ação central seja negada com maior frequência nos casos onde haja a solicitação do paciente.

Este estudo tem algumas limitações. Não é possível comparar as prescrições dos psicotrópicos em relação ao volume de outras prescrições, como realizado em estudos prévios ${ }^{5,10,12}$. Por isso, não é possível afirmar se a prescrição desses medicamentos por cirurgiões-dentistas estão corretas ou estão sendo exageradas. Em segundo lugar, este estudo trabalha com o relato dos cirurgiões-dentistas e não com a prescrição em si, então não há como afirmar se as prescrições foram corretas para cada caso e/ou excessivas. Não conhecer o teor da Portaria 344/1998 não significa que o profissional está prescrevendo de maneira equivocada a medicação psicotrópica, mas demonstra que seu desconhecimento sobre o aspecto legal da prescrição pode comprometer a qualidade do seu atendimento.

Lino et $a .^{5}$ chamam a atenção para a prescrição dessas medicações por caixas e não de forma fracionada. Na odontologia, as doses necessárias para a execução do procedimento clínico são pequenas, e o paciente, ao comprar a caixa, fica com o excedente em casa desse tipo de medicamentos, correndo o risco de utilizá-los fora da indicação correta.

Conclui-se que ser especialista e conhecer a Portaria 344/1998 são fatores associados à prescrição de medicamentos psicotrópicos entre cirurgiões-dentistas.

Mais estudos sobre o tema devem ser estimulados, a fim de que se encontrem estratégias para difundir o conhecimento que permita a utilização desses medicamentos de forma racional e consciente.

\section{REFERÊNCIAS}

1. World Health Organization. As burden of mental disorder looms large, countries report lack of mental health programs: press release WHO/18. Genebra: WHO; 2001 [citado em 2017 Out 26]. Disponível em: www.who.int/ world-health-day/previous/2001/files/whd2001_press_release_en.pdf?ua=1

2. Brasil. Ministério da Saúde. Secretaria de Vigilância Sanitária. Portaria $n^{\circ} 344$, de 12 de maio de 1998. Aprova o Regulamento Técnico sobre substâncias e medicamentos sujeitos a controle especial. Diário Oficial da União [Internet], Brasília, 19 de maio de 1998 [citado em 2017 Out 26]. Disponível em: www.anvisa.gov.br/hotsite/talidomida/legis/Portaria_344_98. pdf

3. Brahm CO, Lundgren J, Carlsson SG, Nilsson P, Hultqvist J, Hagglin C. Dentists' skills with fearful patients: education and treatment. Eur J Oral Sci. 2013;121(3):283-91. http://dx.doi.org/10.1111/eos.12017. PMid:23659263.

4. Okunseri C, Okunseri E, Xiang Q, Thorpe JM, Szabo A. Prescription of opioid and nonopioid analgesics for dental care in emergency departments: findings from the National Hospital Ambulatory Medical Care Survey. J Public Health Dent. 2014;74(4):283-92. http://dx.doi.org/10.1111/jphd.12055. PMid:24863407.
5. Lino PA, Martins MAP, Silva MES, Abreu MHNG. Anxiolytics, sedatives and hypnotics prescribed by dentists in Brazil, 2010. BioMed Res Int. 2017;2017:2841549. http://dx.doi.org/10.1155/2017/2841549. PMid:28638826.

6. Dourado DC, Gonçalves EFS, Melo RO Fo, Poltronieri LC, Dourado VC, Frigo L. Treatment of chronic pain in dentistry using anticonvulsants. Rev Gaucha Odontol. 2016;64(4):447-52. http://dx.doi.org/10.1590/19818637201600030000133236 .

7. Hersh EV, Balasubramaniam R, Pinto A. Pharmacologic management of temporomandibular disorders. Oral Maxillofac Surg Clin North Am. 2008;20(2):197-210, vi. http://dx.doi.org/10.1016/j.coms.2007.12.005. PMid:18343325.

8. Rangel RMR, Roque ICM, Gouvêa CVD, Roque CDM, Martinez OER. Os fármacos na etiologia e tratamento do bruxismo. Rev Bras Ciênc Saúde. 2011;14(4):91-6.

9. Spanemberg JC, Campillo ERR, Salas EJ, López JL. Burning Mouth Syndrome: update. J Dent. 2014;2(2):10-8. http://dx.doi.org/10.12974/23118695.2014.02.01.2. PMid:24984658. 
10. Karki AJ, Holyfield G, Thomas D. Dental prescribing in Wales and associated public health issues. Br Dent J. 2011;210(1):E21. http://dx.doi.org/10.1038/ sj.bdj.2010.1179. PMid:21164522.

11. Moore PA, Nahouraii HS, Zovko JG, Wisniewski SR. Dental therapeutic practice patterns in the U.S. II. Analgesics, corticosteroids, and antibiotics. Gen Dent. 2006;54(3):201-7. PMid:16776415.

12. Hollingworth SA, Chan R, Pham J, Shi S, Ford PF. Prescribing patterns of analgesics and other medicines by dental practitioners in Australia from 2001 to 2012. Community Dent Oral Epidemiol. 2017;45(4):303. http:// dx.doi.org/10.1111/cdoe.12291. PMid:28251672.

13. Rasubala L, Pernapati L, Velasquez X, Burk J, Ren YF. Impact of a mandatory Prescription Drug Monitoring Program on prescription of opioid analgesics by dentists. PLoS One. 2015;10(8):e0135957. http://dx.doi.org/10.1371/ journal.pone.0135957. PMid:26274819.
14. Fox TR, Li J, Stevens S, Tippie T. A performance improvement prescribing guideline reduces opioid prescriptions for emergency department dental pain patients. Ann Emerg Med. 2013;62(3):237-40. http://dx.doi.org/10.1016/j. annemergmed.2012.11.020. PMid:23374416.

15. Centers for Disease Control and Prevention. EPI Info TM7. Version 7.1.4 [Internet]. Atlanta: Centers for Disease Control and Prevention; 2014 [citado em 2017 Out 26]. Disponível em: http://www.cdc.gov/epiinfo

16. Souza GFM, Silva KFFB, Brito ARM. Prescrição medicamentosa em odontologia: normas e condutas. Cad. Saúde Coletiva. 2011;19(2):208-14.

17. Sano PY, Masotti RR, Santos AAC, Cordeiro JA. Avaliação do nível de compreensão da prescrição pediátrica. J Pediatr. 2002;78(2):140-5.

Recebido em: Jan. 08, 2018 Aprovado em: Jan. 03, 2019 